\title{
Acceptabilité et faisabilité de consultations de prévention réalisées par des externes en cabinet de médecine générale. Études CARE-Prev1 \& CARE-Prev2
}

\section{Acceptability and feasibility of prevention consultations carried out by medical students in general practice consulting-room. CARE-Prev1 \& CARE-Prev2 studies}

Julien Gelly ${ }^{1}$, Jean-Pierre AUberT ${ }^{1}$, Dominique Huas ${ }^{1}$, Alain EDDI ${ }^{1}$, Laurence BAUMANN ${ }^{1}$, Sylvie LEGRAIN ${ }^{2}$ et Michel NougaIRÈDE ${ }^{1}$

1 Département de médecine générale, Université Paris 7, France

2 Service de gériatrie, Hôpital Bichat, Paris $18^{\mathrm{e}}$, France

Manuscrit reçu le 30 avril 2008 ; commentaires éditoriaux formulés aux auteurs le 3 juillet 2009 ; accepté pour publication le 15 septembre 2009

\section{Mots clés :}

Médecine générale ; prévention; étudiant en médecine; acceptabilité ; faisabilité
Résumé - Introduction : Le concept des consultations ambulatoires réalisées par des externes et dédiées à la prévention (CARE-Prev) réunit de manière inédite deux sujets d'actualité : la mise en place de stages d'externat de médecine générale et la réalisation de consultations de prévention dédiées en médecine générale. Methode : L'étude CARE-Prev1 a évalué leur acceptabilité par les patients, à l'aide de 494 questionnaires auto-administrés remis aux patients dans deux cabinets de médecine générale. L'étude CARE-Prev2 a évalué leur faisabilité par les externes lors d'une expérience préliminaire, avec comme support un guide de recommandations élaboré à cette occasion. Resultats : Ces consultations ont été jugées acceptables par 76,6\% des patients, sans différence significative par rapport à des consultations dites « classiques ». Ils les ont jugées plus profitables à la formation de l'externe $(86,4 \%)$ qu'à leur propre santé $(64,6 \%)$. À la suite des 18 consultations expérimentales réalisées par 2 étudiantes de $4^{\mathrm{e}}$ année de second cycle, les patients et les externes ayant participé ont émis un avis favorable et espèrent que cette expérience se reproduira. Ils ont suggéré certaines améliorations : organisation du cabinet, respect du libre-choix du patient, optimisation du guide de recommandations. Discussion : Ces deux études ont montré que des consultations de prévention réalisées en médecine générale par des externes étaient acceptables par les patients et que les externes les trouvent faisables par eux et pertinentes pour leur formation. Des études ultérieures seront nécessaires pour étudier leur impact sur la santé des patients ou la formation des externes. 


$\begin{array}{ll}\text { Keywords: } & \text { Abstract - Introduction: The concept of ambulatory consultations performed by medical } \\ \text { General practice; } & \text { students before graduation in general practice dedicated to prevention (CARE-prev) meets } \\ \text { prevention; } & \text { two topical issues: to implement general practice clerkship training and to carry out con- } \\ \text { medical students; } & \text { sultations based on prevention in general practice. Methods: The CARE-Prev1 study has } \\ \text { acceptability; } & \text { evaluated the patients' acceptability from 494 self-questionnaires distributed in two general } \\ \text { feasibility } & \text { practice consulting-rooms. The CARE-Prev2 study has evaluated medical students' feasibility } \\ & \text { during a preliminary experiment, with the support of a recommendation guidebook especially } \\ & \text { elaborated for the study. Results: These consultations were considered acceptable by 76.6\% } \\ & \text { of patients with no significant difference as compare with more "classical" consultations. Pa- } \\ & \text { tients have estimated them more beneficial for medical students training (86.4\%) instead of } \\ & \text { their own health benefit (64.6\%). After 18 experimental consultations carried out by two 6th } \\ & \text { year medical students, patients and medical students mostly had a positive opinion, hoping } \\ & \text { to repeat this experience. They suggested some improvements such as: consulting-room or- } \\ & \text { ganization, respect of patient's free will and optimization of the recommendation guidebook. } \\ & \text { Discussion: Those two studies yielded patients' acceptability of such consultations as well } \\ & \text { as medical students' feasibility and their relevance for their training. Others studies should be } \\ & \text { addressed to assess their outcome on patient's health or on student's training. }\end{array}$

\section{Introduction}

En France, au cours du second cycle des études médicales, les objectifs pédagogiques accordent une place de choix à l'enseignement de la prévention. Depuis l'arrêté du 4 mars $1997^{[1]}$, la validation de ce second cycle est aussi en principe subordonnée à la réalisation d'un stage d'externat en médecine générale. Cependant, cette exigence réglementaire n'est pas encore complètement effective et la plupart des étudiants français continuent d'effectuer leur choix de spécialisation en fin de second cycle (sixième année d'études) sans avoir eu de contact avec la médecine générale.

Parallèlement, une réflexion sur l'organisation de consultations de prévention en médecine générale se développe : la récente réforme du dispositif de l'assurance-maladie ${ }^{[2,3]}$, trois rapports ministériels rendus en 2005 et $2006^{[4-6]}$ et deux avenants à la convention nationale entre les caisses d'assurances maladie et les médecins ${ }^{[7,8]}$ sont les témoins de cette évolution. De plus, une étude réalisée en $2005^{[9]}$ a évalué l'acceptabilité et la faisabilité de consultations de prévention par 60 médecins généralistes à l'aide du logiciel EsPeR (Estimation Personnalisée du Risque) : 498 consultations on été réalisées par 53 médecins généralistes pendant les quatre mois de l'étude; la durée moyenne des consultations a été de $35 \mathrm{~min}$; chaque consultation a permis d'aborder entre trois et six thèmes de prévention dans le champ des maladies cardiovasculaires, de la consommation d'alcool et de tabac et les cancers; 260 patients (52\%) ont émis une opinion favorable et un des points les plus appréciés a été la longueur inhabituelle de la consultation; 92 patients $(18 \%)$ ont été déçus par l'impact insuffisant de ces consultations ou n'ont pas apprécié les explications sur certains risques.

La mise en place de consultations dédiées à la prévention et réalisées par des externes en cabinet de médecine générale permettrait de concilier cette double approche, pédagogique et de santé publique ; elle pourrait présenter de multiples intérêts pour la formation des externes, la santé des patients ou la valorisation de la filière de médecine générale.

Si certaines sociétés savantes éditent déjà des guides de recommandations pour l'examen médical 
périodique $^{[10]}$ et si de nombreuses études soulignent l'intérêt de l'enseignement des pratiques préventives tout au long du cursus des études médicales ${ }^{[11,12]}$, aucune donnée disponible dans la littérature ne concerne la réalisation par des externes de consultations dédiées à la prévention en médecine générale.

Un tel dispositif étant pour l'instant inédit, nous rapportons les résultats de deux études successives, CARE-Prev1 et CARE-Prev2, qui ont évalué d'une part, l'acceptabilité par les patients de telles consultations et, d'autre part, la faisabilité par les externes à l'occasion d'une expérience préliminaire.

\section{Matériels et méthodes}

\section{Matériels}

\section{Élaboration des questionnaires}

La revue de la littérature ne nous a pas permis d'identifier des questionnaires adaptés aux objectifs respectifs de ces deux études, déjà publiés et validés. Des questionnaires originaux ont donc été élaborés sur la base d'un consensus des auteurs de ce travail : les questions portant sur l'acceptabilité et la faisabilité appelaient des réponses formulées à l'aide d'échelles ordinales de type Likert, les autres questions étaient pour la plupart fermées (de type binaire ou à choix multiples) et certaines ouvertes (commentaires libres). Au total, quatre questionnaires auto-administrés ont été élaborés : le premier destiné aux patients de l'étude CARE-Prev1 et les trois autres respectivement aux patients, aux externes et aux praticiens ayant participé à l'étude CARE-Prev2.

\section{Élaboration d'un guide de recommandations}

Avant de proposer de telles consultations de prévention aux patients, l'étape suivante a été de constituer un guide, destiné aux externes qui allaient les réaliser, synthétisant les recommandations actuelles en matière de prévention. Celui-ci devait traiter des thèmes de santé publique prévalents en soins primaires, reposer sur des sources fiables et validées et contenir le cas échéant des outils à utiliser avec le patient pendant la consultation.

Un guide de recommandations de 64 pages a donc été créé à partir d'une recherche bibliographique méthodique. La plupart des références retenues émanaient de bases de données francophones (Haute autorité de santé, Institut national de prévention et d'éducation pour la santé, par exemple), auxquelles ont été ajoutés des textes de littérature secondaire (La revue Prescrire, notamment).

Ce guide était constitué de six chapitres : les conduites addictives (tabac, alcool, cannabis et héroïne); la sexualité (infections sexuellement transmissibles, contraception, interruption volontaire de grossesse, désir de grossesse, grossesse, allaitement, frottis cervico-vaginaux); le risque cardiovasculaire (calcul du risque cardiovasculaire global, hypertension artérielle, dyslipidémies, diabète) ; les cancers (sein, prostate, colon et rectum); les vaccins; les âges de la vie (thèmes de prévention propres aux enfants et aux personnes âgées).

Chaque sous-chapitre traitait d'un thème de prévention et était constitué de quatre parties : données épidémiologiques, recommandations disponibles, outils utilisables en consultation (tels que certains calculs de scores ou algorithmes décisionnels) et références citées (la plupart accessibles par un lien hypertexte).

\section{Déroulement des consultations de prévention}

Durant la première partie de la consultation (15 à 30 minutes), l'externe avait pour consigne de relever les antécédents et facteurs de risque du patient par un interrogatoire approfondi et un examen clinique sommaire (mesure de la tension artérielle, mesure du périmètre abdominal, du poids et de la taille ; calcul de l'indice de masse corporelle). Toutes ces informations étaient colligées dans un document de synthèse, élaboré de manière à mettre en évidence certains facteurs de risque (lorsqu'il existait des données disponibles dans la littérature).

La deuxième partie de la consultation (15 à 30 minutes) était consacrée à la prévention 
proprement dite : l'externe choisissait librement un ou plusieurs thème(s) de prévention qu'elle estimait adapté(s) au patient et mettait en pratique les recommandations contenues dans le guide s'y rapportant. Les externes n'étant pas directement supervisées par un maître de stage au cours de ces consultations, elles ne pouvaient prescrire ni traitement, ni exploration complémentaire. Si elles le jugeaient nécessaire, les externes pouvaient inviter le patient à consulter son médecin traitant. Avec l'accord du patient, les informations recueillies au cours de cette consultation et les propositions de prise en charge étaient transmises au médecin.

Ces consultations étaient gratuites et ne devaient pas durer plus d'une heure.

\section{Méthodes}

\section{Étude CARE-Prevl}

L'étude CARE-Prev1 était une enquête d'opinion transversale, dont l'objectif a été d'évaluer l'acceptabilité par les patients de consultations de prévention réalisées par des externes, si elles devaient à l'avenir être mises en place.

Les questionnaires ont été distribués sur deux sites : un cabinet médical urbain (Paris) dans lequel exerçaient quatre médecins (dont trois maîtres de stage) et un cabinet semi-rural (Vauréal, Val d'Oise) dans lequel exerçaient quatre médecins (dont trois maîtres de stage).

Tous les patients qui sont venus consulter dans ces deux cabinets médicaux pendant quatre semaines consécutives de l'été 2007 et qui ont accepté de répondre au questionnaire ont été inclus dans l'étude. Les critères d'exclusion étaient le refus ou l'incapacité du patient à remplir le questionnaire (y compris avec aide). Les parents pouvaient remplir le questionnaire à la place de leur enfant, s'il n'en était pas capable. Au total, les questionnaires ont été proposés à plus de 700 patients. La distribution des questionnaires était assurée par les secrétaires médicales, avant la consultation, et était précédée d'une courte explication relative aux objectifs de l'étude et aux fonctions de maître de stage, d'externe et d'interne. Les premières questions caractérisaient les patients selon leur âge et leur sexe. Les questions suivantes portaient sur l'acceptabilité de consultations dites «classiques » en présence du médecin habituel et de l'externe (quel que soit celui qui menait la consultation), et de consultations de prévention réalisées uniquement par des externes.

\section{Étude CARE-Prev2}

L'étude CARE-Prev2 était une étude qualitative par expérimentation en situation, dont les objectifs ont été d'évaluer la faisabilité de consultations de prévention par les externes, la satisfaction des acteurs et les difficultés rencontrées.

Deux étudiantes de quatrième année de second cycle ont accepté de participer à cette étude. Elles ont suivi trois heures de formation initiale sur l'utilisation du guide de recommandations (dont elles ont reçu un exemplaire imprimé et relié) et sur le déroulement des consultations.

Les consultations ont été réalisées dans le cabinet de Vauréal pendant quatre jours. Tous les patients ayant consulté au cabinet dans la semaine précédente ont été invités à participer à une consultation de prévention sur rendez-vous; les consultations sans rendez-vous étaient proposées aux patients de la salle d'attente, avant ou après le rendez-vous avec leur médecin.

$\mathrm{Au}$ décours de chaque consultation de prévention, un questionnaire était remis au patient et, à la fin de l'expérimentation, deux questionnaires destinés respectivement aux externes et aux praticiens ont recueilli leur avis.

L'analyse des données a été réalisée avec à la version 3.4.1 du logiciel Epi Info ${ }^{\circledR}$.

\section{Résultats}

L'étude CARE-Prev1 a permis de recueillir 494 questionnaires complétés : 276 par les patients de Vauréal et 218 par ceux de Paris. Les deux tiers des répondants étaient des femmes $(65,8 \%)$, sans différence significative entre les deux sites. Les 


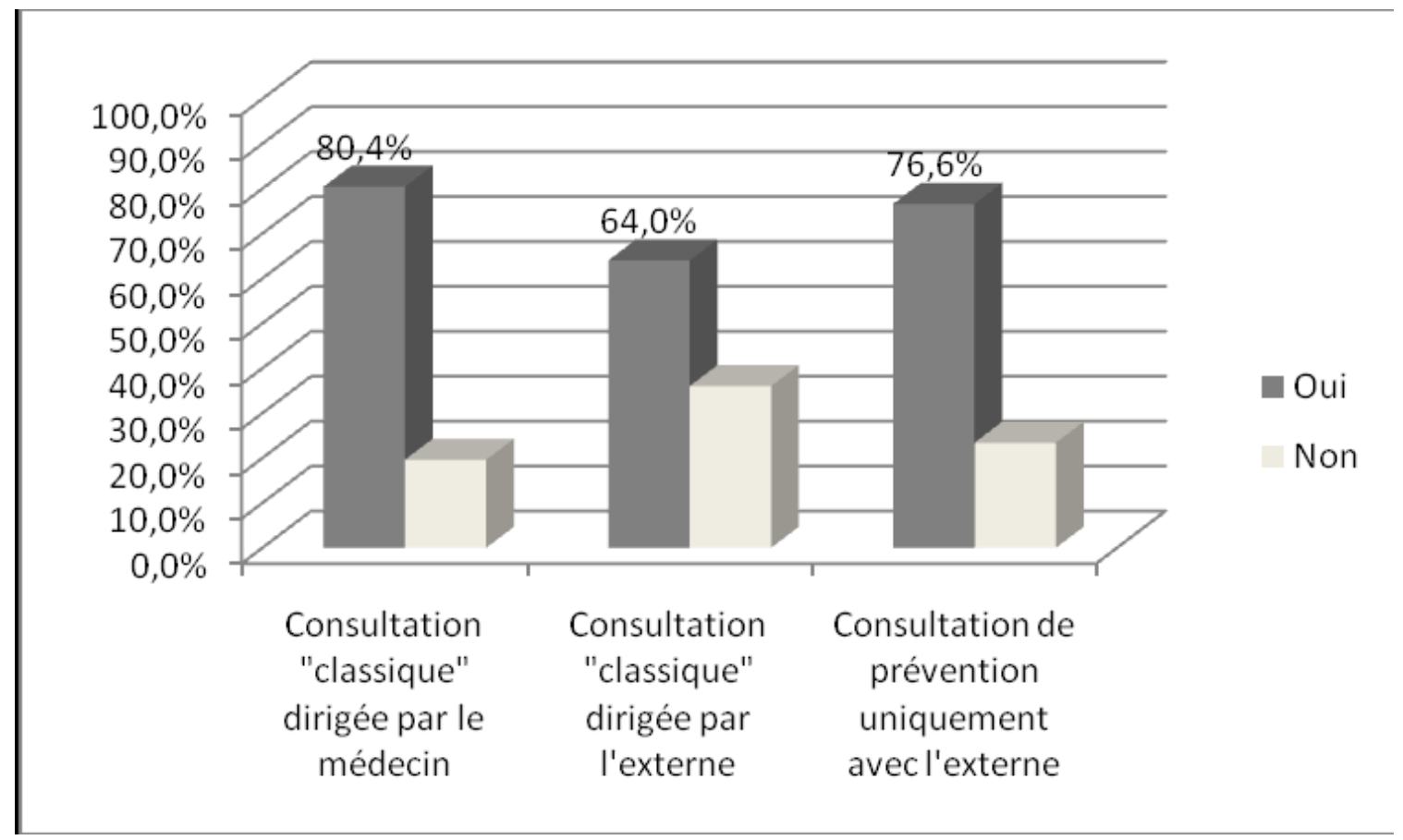

Fig. 1. Acceptabilité des consultations en présence d'un externe. Les histogrammes rendent compte : a) des proportions de patients se déclarant prêts à être reçus en consultation dite « classique » en présence de l'externe selon que, respectivement, le médecin ou l'externe dirige la consultation (colonnes de gauche et du milieu) et; b) des proportions de patients se déclarant prêts à être reçus en consultation de prévention, uniquement en présence de l'externe (colonne de droite).

répondants de Vauréal étaient significativement plus jeunes : les patients de 20 à 30 ans y étaient trois fois plus représentés $(p<0,001$; Odds ratio $=3,55$ avec IC95 \% $=[2,18 ; 5,79])$ et il n'y avait aucun patient de plus de 70 ans.

L'acceptabilité des consultations de la part des patients est globalement bonne (figure 1). Concernant les consultations dites «classiques », les patients déclarent très majoritairement $(80,4 \%)$ accepter d'être reçus par leur médecin en présence d'un externe, sans différence significative entre les deux cabinets. Ils sont tous plus réticents à l'idée que l'externe dirige la consultation, surtout dans le cabinet de Paris $(51,8 \%$ contre $71,4 \% ; p<0,001)$. Les motifs qui représenteraient un frein pour accepter ces consultations en binôme sont ceux touchant à la sexualité ou d'ordre psychologique et, dans une moindre mesure, ceux jugés par les patients comme complexes ou graves (surtout dans l'éventualité où l'externe dirige la consultation).
Concernant les consultations de prévention, les patients des deux sites accepteraient majoritairement d'être reçus uniquement par un externe (76,6\%), sans différence significative par rapport à une consultation dite "classique ». Ils préfèreraient que ces consultations leur soient proposées avant le rendez-vous avec leur médecin ou bien un autre jour avec l'externe mais sur rendez-vous. Une majorité des patients estimait que ce type de consultation pourrait profiter aux externes $(86,4 \%)$ ou à eux-même (64,6 \%) mais ils n'étaient que 39,9\% à penser qu'elles puissent profiter à leur médecin.

L'analyse des réponses ouvertes montre que la plupart des commentaires des patients concernaient directement l'intérêt du contact avec la médecine générale dans la formation de l'externe, ou la confiance que les patients étaient prêts à leur accorder. Certains patients ont regretté que de telles initiatives n'aient pas vu le jour plus tôt. Plusieurs commentaires de patients concernaient l'information et 
la liberté de choix de la procédure de consultation : nécessité d'informer les patients de la présence de l'externe, de laisser le choix d'accepter ou de refuser sa présence et de respecter le secret médical. D'autres ont témoigné d'une certaine méfiance envers les compétences de l'externe.

Au cours de l'étude CARE-Prev2, une consultation de prévention a été proposée à 50 patients : neuf ont accepté de participer le jour-même et 16 ont accepté d'y participer un autre jour avec un rendezvous. Les refus étaient justifiés par le manque de temps ou d'envie. Parmi les 16 patients ayant pris rendez-vous, neuf ne sont pas venus, sept d'entre eux n'ayant pas prévenu le cabinet médical de leur absence. Dans ce cas, il était proposé aux patients en salle d'attente de participer à une consultation de prévention avec l'externe, juste après le rendez-vous avec leur médecin : deux patients ont ainsi été inclus.

Les deux externes ont donc réalisé des consultations de prévention pour 18 patients : cinq hommes (âge médian : 33 ans), huit femmes (âge médian : 35 ans) dont une femme enceinte, et cinq enfants (de cinq mois à 14 ans). Parmi ces 18 participants, 12 ont renvoyé le questionnaire d'évaluation (dont un était inexploitable) : six ont vu l'externe après la consultation avec leur médecin, six autres l'ont vu un autre jour sur rendez-vous. Leur impression globale était «très positive » dans quatre cas et «plutôt positive » dans sept cas. Les points positifs notés par les patients étaient : l'importance du dialogue et de l'écoute; la gratuité et la durée de la consultation; la qualité et la spécificité du contenu (prévention); la complémentarité avec une consultation classique; le respect de la confidentialité et l'absence de risque ressenti. Des points négatifs ont été relevés par cinq patients : le manque d'expérience des externes, le manque d'utilité pour leur santé ou le manque de souplesse dans les horaires proposés. Tous les répondants pensaient que de telles consultations de prévention peuvent profiter aux externes; dix d'entre eux les estiment profitables aux patients et quatre aux maîtres de stage.

L'impression générale des deux externes a été très positive. Elles pensaient que ces consultations de prévention étaient profitables à l'externe et aux patients. Elles auraient aimé recevoir ce type de formation durant leur externat. Elles pensaient que cela pourrait donner le goût de la médecine générale aux externes. Les points positifs qu'elles ont retenus sont : le premier contact avec la médecine générale, la durée inhabituelle de la consultation (20 à 40 minutes) et l'autonomie qui valorise leurs connaissances. Les points négatifs sont en rapport avec les supports peu adaptés (guide de recommandations et document de synthèse élaborés à l'occasion de cette étude), leur déficit de connaissances dans certains domaines (notamment pour la prévention chez l'enfant) ou l'absence de légitimité ressentie vis-à-vis des patients. Enfin, elles ont regretté les rendez-vous manqués et non annulés.

Deux praticiens sur quatre ont répondu au questionnaire. Ils ont jugé cette expérience respectivement « très positive » et «plutôt positive » et ont rappelé la nécessité de la poursuivre pour en évaluer l'impact sur la santé des patients ou sur la formation des externes.

\section{Discussion}

Le travail rapporté présente plusieurs limites.

Il n'existait pas de questionnaire standardisé validé permettant de répondre aux objectifs de ces deux études. À défaut, nous avons donc élaboré des questionnaires originaux. En l'absence de démonstration de leurs qualités métrologiques, le recours à l'outil statistique pour en interpréter les données est cependant discutable. Nos résultats doivent donc être considérés comme résultant d'une étude de nature très exploratoire. Dans une telle perspective, d'autres options méthodologiques auraient d'ailleurs pu être envisagées. Une approche qualitative, recourant par exemple à des entretiens semi-structurés ou à des groupes de discussion focalisée (focus group) aurait probablement été pertinente.

Par ailleurs, l'étude CARE-Prev1 s'est déroulée pendant les vacances scolaires, de sorte que les consultants étaient probablement différents de ceux 
du reste de l'année, notamment pour ceux consultant exceptionnellement dans ces cabinets du fait des congés de leur médecin. Aucun des répondants n'avait plus de 70 ans dans le cabinet de Vauréal. En 2006, cette tranche de la population ne représentait que 1 à $2 \%$ des consultants d'après les relevés individuels d'activité et de prescriptions des médecins. Vauréal est une ville nouvelle où vivent peu de personnes âgées. Dans chacun des cabinets, trois médecins sur quatre étaient maîtres de stage depuis des années. Leurs patients étaient peut-être plus naturellement disposés à être reçus en consultation par des externes. Du fait de sa conception, cette étude ne fournit aucune information sur les non-répondants (âge et sexe, notamment). Elle n'évalue pas si les patients acceptant les consultations de prévention sont ceux chez qui elles seraient le plus bénéfique (absence de recueil de renseignements médicaux relatif au terrain ou aux antécédents).

Compte tenu de sa nature purement descriptive, l'étude CARE-Prev2 ne peut être interprétée en termes d'impact sur la santé des patients ou sur la qualité de la formation des externes, données qui pourraient faire l'objet d'études complémentaires.

En dépit de ces limites, ces études apportent cependant des informations utiles dans le contexte actuel d'un développement progressif du stage d'externat en médecine générale en milieu académique français. Concernant les consultations en présence du médecin et de l'externe, les obstacles les plus souvent cités par les patients correspondent à ceux observés empiriquement par de nombreux internes en stage de médecine générale. Dans l'éventualité où l'externe dirigerait cette consultation, les motifs considérés comme complexes ou graves sont plus souvent cités, ce qui peut traduire un manque de confiance des patients dans les capacités de l'externe.

Pour reproduire cette expérience, les cabinets devraient disposer pendant une journée d'une salle de consultation avec accès aux dossiers médicaux (idéalement informatisés), d'une table d'examen, d'un brassard à tension, d'une toise et d'un pèsepersonne. La fréquence des rendez-vous manqués était peut-être associée au statut de l'externe, qui n'était pas clairement identifié par les patients comme un praticien en formation. Pour les éviter, le médecin devrait peut-être proposer lui-même aux patients de participer aux consultations de prévention.

Cette étude a aussi permis d'apporter certaines améliorations au guide de consultation, au document de synthèse ou à l'organisation des consultations de prévention : adaptation du guide à l'enfant, développement de thèmes sur l'alimentation ou la iatrogénie, fourniture d'autres outils. Enfin, s'agissant simplement d'une expérience de sensibilisation, les consultations de prévention réalisées par les externes n'ont pas été suffisamment exploitées comme de véritables activités de formation (absence de rétroaction explicite et structurée ou de supervision différée, notamment).

\section{Conclusion}

Les études CARE-Prev1 et CARE-Prev2 ont montré que des consultations de prévention réalisées en médecine générale par des externes étaient acceptables par les patients et que les externes les trouvent faisables par eux et pertinentes pour leur formation. Si l'ensemble de la démarche préventive ne peut évidemment pas reposer sur le seul externe, ces consultations de prévention pourraient se tenir une fois par semaine, de manière à les sensibiliser à un des aspects essentiels de la médecine de soins primaires. Des études ultérieures seront nécessaires pour démontrer que de telles consultations sont bénéfiques pour la santé des patients ou la formation des externes. Idéalement, ces consultations de prévention devraient s'intégrer aux stages d'externat en médecine générale. Dans une telle perspective, il serait important de pouvoir évaluer l'impact de ce type de stage (et de ce type de dispositif pédagogique en particulier) auprès de l'ensemble des étudiants en médecine. 


\section{Contributions}

Julien Gelly a participé à la conception du protocole de recherche, au recueil des données, à l'interprétation des résultats, à l'analyse statistique et à l'écriture du manuscrit. Jean-Pierre Aubert a participé à la conception du protocole de recherche, à l'interprétation des résultats et à l'écriture du manuscrit. Dominique Huas, Alain Eddi et Laurence Baumann et Michel Nougarède ont paricipé à l'écriture du manuscrit. Sylvie Legrain a participé à la conception du protocole de recherche et à l'interprétation des résultats.

Ce travail a été élaboré dans le cadre d'un travail académique (thèse pour le diplôme d'état de doctorat en médecine, soutenue par Julien Gelly le 20 mars 2008 à l'université Paris 7, médaille d'argent); il a fait l'objet de deux communications orales, au congrès de médecine générale à Lyon le 14 juin 2008 et au congrès du Collège national des généralistes enseignants à Angers le 28 novembre 2008 , et d'un poster au congrès de la Wonca à Istanbul du 4 au 7 septembre 2008. Je tiens à remercier les deux externes qui ont accepté de réaliser les consultations de prévention, Audrey Fel et Laurenne Lhuillier, ainsi que les patients, les secrétaires et les practiciens des deux cabinets médicaux de cette étude.

\section{Références}

1. Arrêté du 4 mars 1997 relatif à la deuxième partie du deuxième cycle des études médicales. NOR: MENU9700546A. Journal Officiel de la République Française ; n 72 du 26 mars 1997 : 4684. [On-line] Disponible sur : http://www. legifrance.gouv.fr/ WAspad/UnTexteDeJorf?numjo=MENU9700546A

2. Loi n²004-806 du 9 août 2004 relative à la politique de santé publique. NOR: SANX0300055L. Journal Officiel de la République Française ; n ${ }^{\circ} 185$ du 11 août 2004:14277. [on-line] Disponible sur : http://www. legifrance.gouv . fr/WAspad/UnTexteDeJorf? numjo $=$ SANX0300055L

3. Loi n²004-810 du 13 août 2004 relative à l'assurance maladie (rectificatif). NOR: SANX0300055L. Journal Officiel de la République Française ; n²76 du 27 novembre 2004 :20151. [On-line] Disponible sur : http://www.legifrance.gouv.fr/WAspad/ UnTexteDeJorf?numjo=SANX0300055L

4. Commission "Démographie médicale". Rapport présenté par le professeur Yvon Berland 2005. [On-line] Disponible sur : http://www.sante.gouv.fr/htm/ actu/berland_demomed/rapport.pdf

5. La consultation périodique de prévention. Rapport présenté par le professeur Joël Ménard 2005. [On-line] Disponible sur : http://www.sfsp.info/sfsp/ infos/documents/rapportmenard.pdf

6. Stratégies nouvelles de prévention. Rapport de la Commission d'orientation prévention présidée par le Docteur Jean-François Toussaint 2006. [On-line] disponible sur : http://www.sante.gouv.fr/htm/ actu/toussaint/rapport.pdf

7. Arrêté du 23 mars 2006 portant approbation de l'avenant $\mathrm{n}^{\circ} 12$ à la convention nationale des médecins généralistes et des médecins spécialistes. NOR: SANS0621311A. Journal Officiel de la République Française $\mathrm{n}^{\circ} 76$ du 30 mars 2006 :4768. [On-line] Disponible sur : http: //www . legifrance.gouv. fr/WAspad/ UnTexteDeJorf?numjo=SANS0621311A

8. Arrêté du 2 mai 2007 portant approbation de l'avenant n²3 à la convention nationale des médecins généralistes et des médecins spécialistes. NOR: SANS0752329A. Journal Officiel de la République Française ; n¹03 du 3 mai 2007 :7826. [On-line] Disponible sur : http://www . legifrance.gouv.fr/WAspad/ UnTexteDeJorf?numjo=SANS0752329A

9. Chevallier P, Colombet I, Wajs C, Leneveut L, Chatellier $\mathrm{G}$. Une consultation de prévention dédiée et structurée à l'aide d'un outil informatique. Étude C-PRED-EsPeR : nouveau concept pour la vraie vie ? Exercer 2008;81:3641

10. L'examen médical périodique de l'adulte. Données probantes 2009. Direction de santé publique de l'Agence de la santé et des services sociaux de Montréal. Collège des médecins. 2009. [On-line] Disponible sur : http://www . santepub-mtl.qc.ca/ Publication/pdfautre/examenperiodique2009. $\operatorname{pdf}$

11. Frank E, Carrera JS, Elon L, Hertzberg VS. Predictors of US medical students' prevention counseling practices. Prev Med 2007;44:76-81.

12. Martens FM, van der Vleuten CP, Grol RP, op 't Root JM, Crebolder HF, Rethans JJ. Educational objectives and requirements of an undergraduate clerkship in general practice. The outcome of a consensus procedure. Fam Pract. 1997;14:153-9

Correspondance et offprints : Julien GELLY, 1 rue Jean Moulin, 93260 Les Lilas, France

Mailto : juliengelly81@gmail.com 